\title{
RECUENTOS CROMOSOMICOS EN PLANTAS QUE CRECEN EN CHILE. III
}

\section{CHROMOSOMES REPORT FROM PLANTS ARE GROWING IN CHILE. III}

\author{
Carlos M. Baeza ${ }^{1}$, Otto Schrader ${ }^{2}$, Anass Terrab ${ }^{3}$, Tod Stuessy ${ }^{3}$, Marcelo Rosas ${ }^{4}$, \\ Eduardo Ruiz ${ }^{1}$, María Negritto ${ }^{1} \&$ Estrella Urtubey ${ }^{5}$
}

${ }^{1}$ Facultad de Ciencias Naturales y Oceanográficas, Departamento de Botánica, Casilla 160-C, Universidad de Concepción, Concepción, Chile. cbaeza@udec.cl

${ }^{2}$ Federal Centre for Breeding Research on Cultivated Plants, Institute of Horticultural Crops, Neuer Weg 22/23, 06484 Quedlinburg, Germany

${ }^{3}$ Institute of Botany, University of Vienna, Rennweg 14, A-1030 Wien, Austria.

${ }^{4}$ Instituto de Investigaciones Agropecuarias. Camino a Peralillo s/n, Vicuña. Chile.

${ }^{5}$ División Plantas Vasculares, Museo de la Plata, Paseo del Bosque, La Plata, CA. P. 1900, Argentina.

\section{RESUMEN}

Se entregan datos citológicos del número cromosómico diploide de 24 especies de la flora vascular de Chile, utilizándose material de raíces provenientes de plántulas. Nuevos registros para la flora de Chile son: Alstroemeria presliana Herb. subsp. presliana $(2 \mathrm{n}=16)$, Chaetanthera moenchioides Less. $(2 \mathrm{n}=26)$, Hypochaeris scorzonerae (DC.) F.Muell. $(2 \mathrm{n}$ $=8)$, Phycella bicolor (Ruiz et Pav.) Herb. $(2 \mathrm{n}=16)$ y Rhodophiala tiltilensis (Traub et Moldenke) Traub $(2 \mathrm{n}=16)$.

PALABRAS Claves: Números cromosómicos, flora vascular de Chile.

\section{ABSTRACT}

Chromosome numbers of 24 Chilean taxa of vascular plants were studied using root-tip mitotic metaphases. New records for the chilean Flora are: Alstroemeria presliana Herb. subsp. presliana $(2 \mathrm{n}=16)$, Chaetanthera moenchioides Less. $(2 \mathrm{n}=26)$, Hypochaeris scorzonerae (DC.) F.Muell. $(2 \mathrm{n}=8)$, Phycella bicolor (Ruiz et Pav.) Herb. $(2 \mathrm{n}=16)$ and Rhodophiala tiltilensis (Traub et Moldenke) Traub $(2 \mathrm{n}=16)$.

KeYwords: Chromosome numbers, Chilean vascular flora.

\section{INTRODUCCION}

A nivel mundial se conoce el número de cromosomas de alrededor de un $25 \%$ de las Angiospermas (Bennett 1998). La información sobre recuentos cromosómicos en plantas vasculares de Chile es aún más deficitaria (Stuessy \& Taylor 1995). Esta carencia de información dificulta estudios de importancia sobre la sistemática y biodiversidad de grupos determinados (Baeza et al. 2001, 2004). Alrededor de un $45 \%$ de la flora de Chile es endémica (Marticorena 1990), y de ella no hay un conocimiento citológico básico, muchas veces indispensable para lograr una clasificación que refleje relaciones evolutivas. Esta falta de conocimiento impide una adecuada evaluación de la biodiversidad de regiones particulares y por lo tanto dificulta dilucidar el origen de la flora y comprender la estructura y dinámica actual de la vegetación (Arroyo et al. 1993). Este trabajo entrega nueva información sobre recuentos cromosómicos de la flora vascular de Chile. En este reporte se informa sobre el número de cromosomas de 24 especies de plantas vasculares que crecen en Chile. 


\section{MATERIALES Y METODOS}

Puntas de raíces de 1-2 cm de longitud, obtenidas a partir de plántulas germinadas en placas Petri, fueron tratadas con una solución de 8-hidroxiquinoleina ( $2 \mathrm{mM}$ ) por 24 horas a $4^{\circ} \mathrm{C}$ o en Colchicina $0,1 \%$ durante 4 horas, 2 a temperatura ambiente y a la oscuridad y 2 horas a $5{ }^{\circ} \mathrm{C}$. Posteriormente, se fijaron en etanol / ácido acético (3:1) por 24 horas y se almacenaron en alcohol $70 \%$ a $-20{ }^{\circ} \mathrm{C}$. Previo a la maceración, las raíces fueron lavadas 3 veces en agua destilada por 30 minutos, para luego ser digeridas en una mezcla de enzimas de $4 \%$ celulasa "Onozuka R-10" (Serva) y $1 \%$ pectiolasa Y-23 (Seishin Pharmaceutical) en $75 \mathrm{mM}$ de $\mathrm{KCl}$, a pH 4,0 por 40 minutos a $37^{\circ} \mathrm{C}$. Después de un breve lavado en agua destilada, las raíces fueron mantenidas durante un minuto en ácido acético al $45 \%$ y luego se hizo el aplastado correspondiente. Los cubreobjetos fueron removidos después de mantener las muestras a $84^{\circ} \mathrm{C}$. Los preparados se dejaron secar durante 1 2 días a temperatura ambiente y se almacenaron a $20^{\circ} \mathrm{C}$. Los cromosomas fueron teñidos con DAPI (1,0 ng/l de 4',6-diamidino-2-fenilindol). Esta metodología fue utilizada para las siguientes especies: Alstroemeria aurea Graham, A. hookeri Lodd. subsp. hookeri, A. ligtu L., A. pelegrina L., A. presliana Herb. subsp. presliana, Chaetanthera chilensis (Willd.) DC., Ch. ciliata Ruiz et Pav., Ch. microphylla (Cass.) Hook. et Arn., Ch. moenchioides Less., Grindelia anethifolia (Phil.) A.Bartoli et Tortosa, G. prunelloides (Less.) A.Bartoli et Tortosa, Haplopappus glutinosus Cass., H. grindelioides (Less.) DC. y H. stolpii Phil. Para las especies Hypochaeris scorzonerae (DC.) F.Muell., H. radicata L., H. incana (Hook. et Arn.) Macloskie, H. acaulis (J.Remy) Britton, Haplopappus macrocephalus (Poepp. ex Less.) DC., Leontodon autumnalis L., Phycella bicolor (Ruiz et Pav.) Herb., Ph. scarlatina Ravenna y Rhodophiala tiltilensis (Traub et Moldenke) Traub, posterior a la fijación, se realizó una hidrólisis ácida con $\mathrm{HCl} 0,5 \mathrm{~N}$ durante 25 minutos a $40{ }^{\circ} \mathrm{C}$, luego se lavó con agua destilada en 2 oportunidades y por último se tiñó el ápice de la raíz con orceina acética al $1 \%$ y se realizó el aplastado. Las placas metafásicas se fotografiaron en un microscopio Zeiss Axioskop y fueron contados por observación directa en las fotomicrografías. El material de referencia (Anexo 1) está depositado en el Herbario del Departamento de Botánica de la Universidad de Concepción (CONC).

\section{RESULTADOS}

Alstroemeriaceae

Alstroemeria aurea Graham: $2 \mathrm{n}=16$ (Fig. $1 \mathrm{~A}$ ).

Alstroemeria hookeri Lodd. subsp. hookeri: $2 \mathrm{n}=$ 16 (Fig. 1 B).

Alstroemeria ligtu L.: $2 \mathrm{n}=16$ (Fig. $1 \mathrm{C}$ ).

Alstroemeria pelegrina L.: $2 \mathrm{n}=16$ (Fig. $1 \mathrm{D}$ ).

Alstroemeria presliana Herb. subsp. presliana: $2 \mathrm{n}$ $=16$ (Fig. $1 \mathrm{E})$.

AmaryllidaceAe

Phycella bicolor (Ruiz et Pav.) Herb.: $2 \mathrm{n}=16$

(Fig. $1 \mathrm{~T}$ ).

Phycella scarlatina Ravenna: $2 \mathrm{n}=16$ (Fig. $1 \mathrm{U}$ ).

Placea amoena Phil.: $2 \mathrm{n}=16$ (Fig. $1 \mathrm{~V}$ ).

Rhodophiala tiltilensis (Traub et Moldenke) Traub: $2 \mathrm{n}=16$ (Fig. $1 \mathrm{~W}$ ).

Asteraceae

Chaetanthera chilensis (Willd.) DC.: $2 n=22$ (Fig. 1 F).

Chaetanthera ciliata Ruiz et Pav.: 2n=22 (Fig. 1 G).

Chaetanthera microphylla (Cass.) Hook. et Arn.: $2 \mathrm{n}=24$ (Fig. $1 \mathrm{H})$.

Chaetanthera moenchioides Less.: $2 \mathrm{n}=26$

(Fig. 1 I).

Grindelia anethifolia (Phil.) A.Bartoli \& Tortosa: $2 \mathrm{n}=12$ (Fig. $1 \mathrm{~J}$ ).

Grindelia prunelloides (Less.) A.Bartoli \& Tortosa: 2n = 12 (Fig. $1 \mathrm{~K}$ ).

Haplopappus glutinosus Cass.: 2n = 10 (Fig. 1 L). Haplopappus grindelioides (Less.) DC.: $2 \mathrm{n}=10$ (Fig. $1 \mathrm{M}$ ).

Haplopappus macrocephalus (Poepp. ex Less.) DC.: $2 \mathrm{n}=10$ (Fig. $1 \mathrm{~N})$.

Haplopappus stolpii Phil.: 2n = 10 (Fig. $1 \tilde{\mathrm{N}}$ ).

Hypochaeris acaulis (J.Remy) Britton: $2 \mathrm{n}=8$ (Fig. 1 O).

Hypochaeris incana (Hook. et Arn.) Macloskie: $2 \mathrm{n}=8$ (Fig. $1 \mathrm{P}$ ).

Hypochaeris radicata $\mathrm{L}$ : $2 \mathrm{n}=8$ (Fig. $1 \mathrm{Q}$ ).

Hypochaeris scorzonerae (DC.) F.Muell.: $2 n=8$ (Fig. 1 R).

Leontodon autumnalis L.: $2 \mathrm{n}=12$ (Fig. $1 \mathrm{~S}$ ). 
Recuentos cromosómicos en plantas que crecen en Chile. III. BAEZA, M. ET AL.
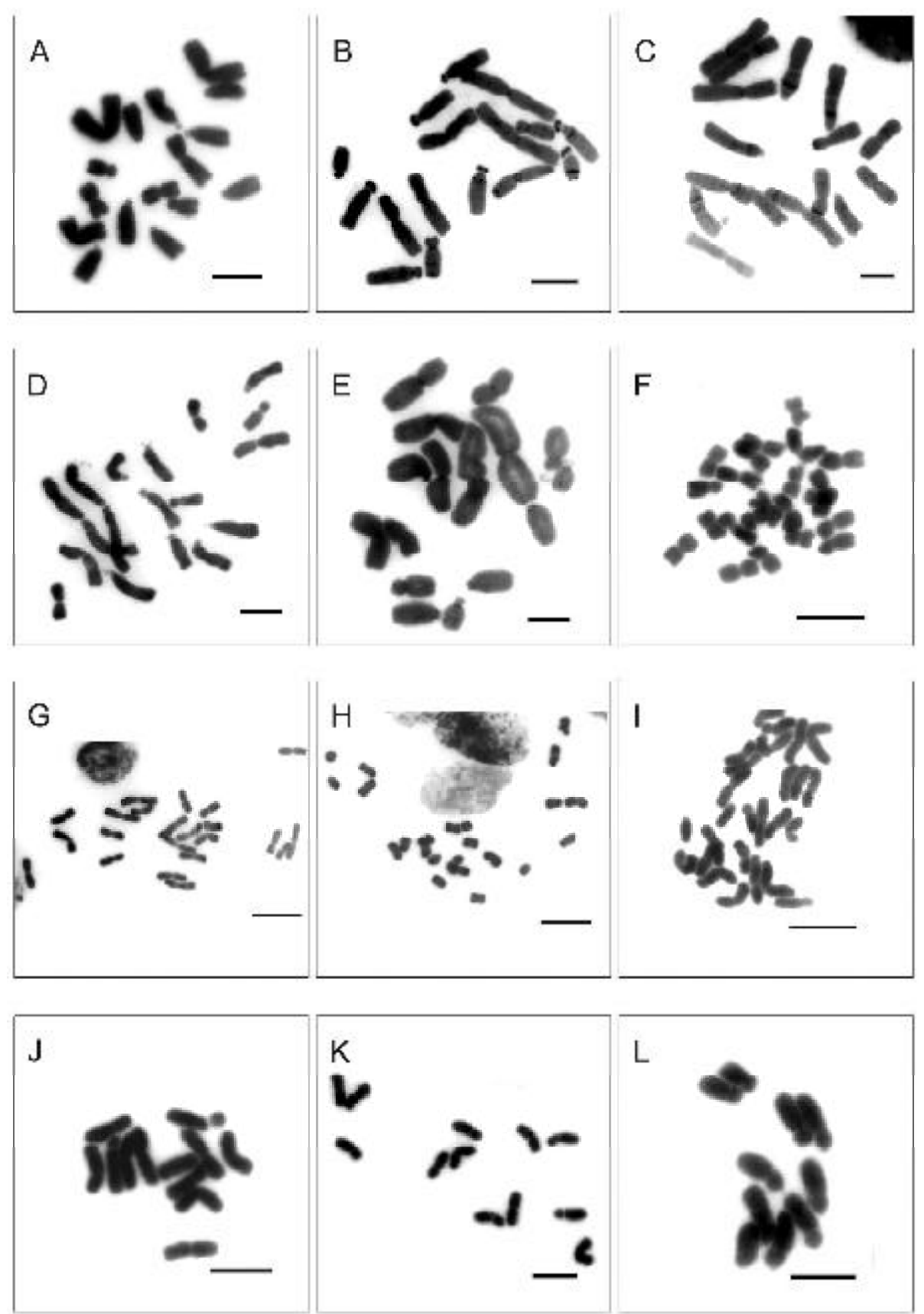

FIGURA 1. Placas metafásicas. A: Alstroemeria aurea $(2 \mathrm{n}=16)$. B: Alstroemeria hookeri subsp. hookeri $(2 \mathrm{n}=16)$. C: Alstroemeria ligtu $(2 \mathrm{n}=16)$. D: Alstroemeria pelegrina $(2 \mathrm{n}=16)$. E: Alstroemeria presliana subsp. presliana $(2 \mathrm{n}=16)$. F: Chaetanthera chilensis. G: Chaetanthera ciliata $(2 \mathrm{n}=22) . \mathrm{H}$ : Chaetanthera microphylla $(2 \mathrm{n}=24)$. I: Chaetanthera moenchioides $(2 \mathrm{n}=26)$. J: Grindelia anethifolia $(2 \mathrm{n}=12)$. K: Grindelia prunelloides $(2 \mathrm{n}=12)$. L: Haplopappus glutinosus $(2 \mathrm{n}=10)$.

Figure 1. Metaphase plates. A: Alstroemeria aurea $(2 \mathrm{n}=16)$. B: Alstroemeria hookeri subsp. hookeri $(2 \mathrm{n}=16)$. C: Alstroemeria ligtu $(2 \mathrm{n}=16)$. D: Alstroemeria pelegrina $(2 \mathrm{n}=16)$. E: Alstroemeria presliana subsp. presliana $(2 \mathrm{n}=16)$. F: Chaetanthera chilensis. G: Chaetanthera ciliata $(2 \mathrm{n}=22)$. H: Chaetanthera microphylla $(2 \mathrm{n}=24)$. I: Chaetanthera moenchioides $(2 \mathrm{n}=26)$. J: Grindelia anethifolia $(2 \mathrm{n}=12)$. K: Grindelia prunelloides $(2 \mathrm{n}=12)$. L: Haplopappus glutinosus $(2 \mathrm{n}=10)$. 


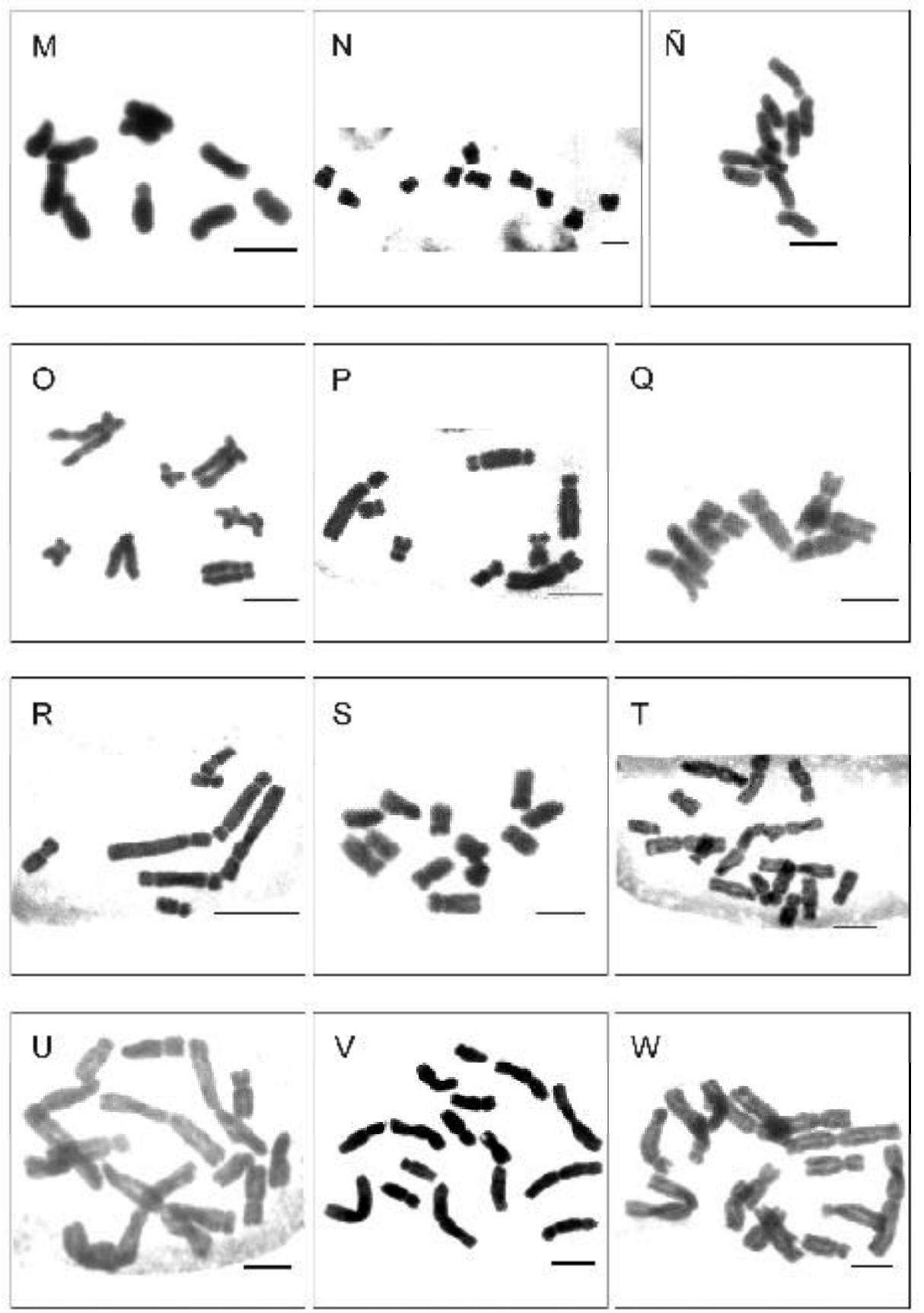

Continuación Figura 1. Placas metafásicas. M: Haplopappus grindelioides $(2 \mathrm{n}=10)$. N: Haplopappus macrocephalus $(2 \mathrm{n}=10)$. N: Haplopappus stolpii $(2 \mathrm{n}=10)$. O: Hypochaeris acaulis $(2 \mathrm{n}=8)$. P: Hypochaeris incana $(2 \mathrm{n}=8) . \mathrm{Q}$ : Hypochaeris radicata $(2 \mathrm{n}=8)$. R: Hypochaeris scorzonerae $(2 \mathrm{n}=8)$. S: Leontodon autumnalis $(2 \mathrm{n}=12)$. T: Phycella bicolor $(2 \mathrm{n}=16)$. U: Phycella scarlatina $(2 \mathrm{n}=16)$. V: Placea amoena $(2 \mathrm{n}=16)$ y W: Rhodophiala tiltilensis $(2 \mathrm{n}=16)$. La barra corresponde a $10 \mu \mathrm{m}(\mathrm{A}-\mathrm{I}, \mathrm{N}, \mathrm{O}-\mathrm{W})$ y $5 \mu \mathrm{m}(\mathrm{J}-\mathrm{M}, \tilde{\mathrm{N}})$.

Continuation Figure 1. Metaphase plates. M: Haplopappus grindelioides $(2 \mathrm{n}=10)$. N: Haplopappus macrocephalus $(2 \mathrm{n}=10)$. $\tilde{\mathrm{N}}$ : Haplopappus stolpii $(2 \mathrm{n}=10)$. O: Hypochaeris acaulis $(2 \mathrm{n}=8)$. P: Hypochaeris incana $(2 \mathrm{n}=8)$. Q: Hypochaeris radicata $(2 \mathrm{n}=8)$. R: Hypochaeris scorzonerae $(2 \mathrm{n}=8)$. S: Leontodon autumnalis $(2 \mathrm{n}=12)$. T: Phycella bicolor $(2 \mathrm{n}=16)$. U: Phycella scarlatina $(2 \mathrm{n}=16)$. V: Placea amoena $(2 \mathrm{n}=16)$ y W: Rhodophiala tiltilensis $(2 \mathrm{n}=16)$. Scale bar $10 \mu \mathrm{m}(\mathrm{A}-\mathrm{I}, \mathrm{N}, \mathrm{O}-\mathrm{W})$ and $5 \mu \mathrm{m}(\mathrm{J}-\mathrm{M}, \tilde{\mathrm{N}})$. 


\section{DISCUSION}

De las 24 especies analizadas, 5 corresponden a nuevos recuentos para la flora chilena:Alstroemeria presliana subsp. presliana $(2 \mathrm{n}=16), C h$. moenchioides $(2 \mathrm{n}=26)$, Hypochaeris scorzonerae $(2 \mathrm{n}=8)$, Phycella bicolor $(2 \mathrm{n}=16)$ y Rhodophiala tiltilensis $(2 \mathrm{n}=16)$.

Alstroemeriaceae: Baeza et al. (2001) indican para Alstroemeria presliana subsp. australis Ehr.Bayer un $2 \mathrm{n}=16$ cromosomas, la misma dotación cromosómica encontrada en A. presliana subsp. presliana. De las restantes especies estudiadas existen recuentos previos que son idénticos a los encontrados en este trabajo: Alstroemeria aurea Graham (Buitendijk \& Ramanna 1996, De Jeu et al. 1997, Buitendijk et al. 1998), A. hookeri (Tsuchiya \& Hang 1989), A. ligtu (Buitendijk et al. 1998; Baeza et al. 2001, Zhouet al. 2003), A. pelegrina (Stephens et al. 1993, Buitendijk et al. 1998).

Amaryllidaceae: Phycella Lindley (Amaryllidaceae) es un género endémico de Chile, constituido por 4 especies (Ravenna 2003). Palma-Rojas (2000) indica para Phycella ignea (Lindl.) Lindl. y Phycella scarlatina $2 \mathrm{n}=16$ y 32 cromosomas, respectivamente. Meerow \& Snijman (1998) señalan 2n = 16 para las especies de Phycella. La especie Phycella bicolor aquí estudiada presenta un $2 n=16$, lo que se corresponde con los reportes anteriores para el género. La especie $P$. scarlatina presentó un $2 \mathrm{n}=16$, a diferencia del tetraploide reportado por Palma-Rojas (2000). Naranjo \& Poggio (2000) realizan un análisis del cariotipo en cinco especies de Rhodophiala de Chile y Argentina, indicando $2 \mathrm{n}=16,18,36,54$ y 72 , dependiendo de las especies. La especie estudiada, Rhodophiala tiltilensis presenta un $2 \mathrm{n}=16$, la misma dotación cromosómica presentada por $R$. bifida (Herb.) Traub, R. andicola (Poepp.) Traub y $R$. rhodolirion (Baker) Traub. Palma-Rojas (2000) reporta el número cromosómico de las especies chilenas Rhodophiala phycelloides (Herb.) Hunz., R. bagnoldii (Herb.) Traub y R. advena (Ker-Gawl.) Traub, todas con un $2 \mathrm{n}=18$, indicando además la presencia de cromosomas supernumerarios. En $R$. tiltilensis no se observó este tipo de cromosomas. Uno de los géneros de Amaryllidaceae chilenos más problemáticos taxonómicamente es Rhodophiala, debido a la gran variación de sus caracteres morfológicos. Es probable que el número cromosómico conjuntamente con caracteres morfológicos constituya una herramienta que podría ayudar consistentemente en la clarificación de muchas especies de posición incierta en este género. Además del número cromosómico, el estudio detallado del cariotipo entrega información muy valiosa que permitirá a futuro no sólo resolver la taxonomía del género sino también de la familia, la que está compuesta por otros géneros tales como Habrantus, Phycella, Placea, Traubia, entre otros, de difícil delimitación entre ellos. El número cromosómico de Placea amoena ya había sido reportado anteriormente (Baeza \& Schrader 2004).

Asteraceae: Grau (1987) reportó el número de cromosomas en algunas especies chilenas de Chaetanthera: Ch. glabrata (DC.) Meigen $(2 \mathrm{n}=$ 28), Ch. linearis Poepp. ex Less. $(2 \mathrm{n}=22)$ y $C h$. linearis var. albiflora Phil. $(2 \mathrm{n}=24)$. Powell et al. (1974) indican un $n=14$ para Ch. tenella Less. Baeza \& Schrader (2005b) reportan para Chaetanthera microphylla $2 \mathrm{n}=24$ cromosomas y Baeza \& Schrader (2005c) indican para Chaetanthera chilensis y Chaetanthera ciliata $2 \mathrm{n}=22$. El nuevo registro que aquí se indica para $C h$. moenchioides, con un $2 \mathrm{n}=26$ constituye un nuevo número cromosómico para este género. Este variado nivel de ploidía en Chaetanthera podría explicar en parte las dificultades taxonómicas reconocidas en este grupo de plantas en Chile.

Existen numerosos recuentos cromosómicos en Hypochaeris, tanto de especies europeas como americanas (Weiss et al. 2003 a y b, Baeza et al. 2004, 2006). H. scorzonerae, especie endémica de Chile, y una de las de mayor tamaño, presenta un $2 \mathrm{n}$ $=8$, igual que el resto de las especies chilenas y americanas.

Leontodon autumnalis es una especie introducida en la XII Región de Chile que cubre grandes extensiones de terreno, sobre todo a orillas de caminos y en sectores anegados (Matthei 1995). Es una maleza común y este reporte cromosómico $2 n=12$ constituye el primero para esta especie en Chile y que se corresponde con los realizados en ejemplares de Europa (Morton 1981, Lovkvist \& Hultgard 1999).

Por último, en Grindelia anethifolia (Bartoli \& Tortosa 1998 a y b; Baeza \& Schrader 2005a), Grindelia prunelloides (Bartoli \& Tortosa 1998a, Baeza \& Schrader 2005a), Haplopappus glutinosus, 
H. grindelioides y H. stolpii (Baeza \& Schrader 2005a), H. macrocephalus (Grau 1976), Hypochaeris incana (Weiss et al. 2003 a) e H. radicata (Baeza et al.2001) hay recuentos cromosómicos previos que se corresponden con los encontrados en este trabajo.

Es importante y necesario indicar que independiente del número de oportunidades que los cromosomas de una especie hayan sido contados nunca está demás investigar la dotación cromosómica en otras poblaciones de la misma especie. Muchas veces, recontar cromosomas en una especie permite establecer el nivel de ploidía preciso de ésta o encontrar cambios en él, que a veces ayudan a entender mejor la variabilidad morfológica de un taxón determinado.

\section{AGRADECIMIENTOS}

Los autores agradecen el apoyo prestado por el proyecto DIUC N ${ }^{\circ} 204.111 .036-1.0$ y a la Fundación Alexander von Humboldt (Georg Foster Stipendium). También se agradece al proyecto BBVA: "Erosión genética durante la radiación evolutiva y su impacto sobre los sistemas reproductores en plantas de América Latina: el género Hypochaeris (Asteraceae) como modelo", proyecto Fundación Andes $N^{\circ} \mathrm{C}-14055$ y al Departamento de Botánica de la Universidad de Concepción por las facilidades otorgadas. También se agradece el apoyo del proyecto Fondecyt $\mathrm{N}^{\circ}$ 1070520.

\section{BIBLIOGRAFIA}

Arroyo, M.T.K., J. Armesto, F. SQueo \& J. Gutiérrez. 1993. Global Change: The flora and vegetation of Chile. En H. Mooney, E. Fuentes \& B. Kronberg (eds). Earth Systems response to Global Change: Contrast between North and South America. Academic Press, New York, pp. 239-264.

Baeza, C. \& O. Schrader. 2004. Karyotype analysis of Placea amoena Phil. (Amaryllidaceae) by double fluorescence in situ hybridization. Caryologia 57: 200-205.

BAEZA, C. \& O. Schrader. 2005a Comparative karyotype analysis in Haplopappus Cass. and Grindelia Willd. (Asteraceae) by double FISH with rRNA specific genes. Plant Systematics and Evolution 251: 161-172.
BAeza, C. \& O. Schrader. 2005b. Análisis del cariotipo y deteccion de los genes $5 \mathrm{~S}$ y $18 \mathrm{~S} / 25 \mathrm{~S}$ rDNA en Chaetanthera microphylla (Cass.) Hook. et Arn. (Asteraceae). Gayana Botánica 62(1): 49-51.

BAEZA, C. \& O. Schrader. 2005c. Karyotype analysis in Chaetanthera chilensis (Willd.) DC. and Chaetanthera ciliata Ruiz et Pavón (Asteraceae) by double fluorescence in situ hybridization. Caryologia 58(4): 332-338.

Baeza, C., G. Kottirsch, J. Espejo \& R. Reinoso. 2001. Recuentos cromosómicos en plantas chilenas. I. Gayana Botánica 58: 133-137.

Baeza, C., E. Vosyka \& T. Stuessy. 2004. Recuentos cromosómicos en plantas que crecen en Chile. II. Darwiniana 42: 25-29.

BAEZA, C., S. J JRA \& T.STUESSY. 2006. Cytogenetic studies in Populations of Hypochaeris a pargioides Hook. et Arn. (Asteraceae, Lactuceae) from Chile. Gayana Botánica 63(1): 99-105.

Bartoli, A. \& R. Tortosa. 1998a. Intermediacy between Grindelia chiloensis and Haplopappus pectinatus (Astereae, Asteraceae). Taxon 47: 337-345.

Bartoli, A. \& R. Tortosa. 1998 b. Estudios cromosómicos en especies sudamericanas de Grindelia (Astereae, Asteraceae). Kurtziana 26: 165-171.

Bennett, M. 1998. Plant genome values: how much do we know?. Proceedings of the Natural Academy of Science, USA 95: 2011-2016.

Buitendij, J. \& M. Ramanna. 1996. Giemsa C-banded karyotypes of eight species of Alstroemeria $\mathrm{L}$. and some of their hybrids. Annals of Botany 78: 449-457.

Buitendijk, J.,A. Peters, R. Quene \& M. Ramanna. 1998. Genome size variation and $\mathrm{C}$ - band variation polymorphism in Alstroemeria aurea, A. ligtu, and A. magnifica (Alstroemeriaceae). Plant Systematics and Evolution 212: 87-106.

De Jeu, M., J. Lasschuit, S. Kuipers \& R. Visser. 1997. Characterization and localization of repetitive DNA sequences in the ornamental Alstroemeria aurea Graham. Theoretical and Applied Genetic 94: 982-990.

GrAU, J. 1976. Chromosomenzahlen von sudamerikanischen Haplopappus-Arten. Mitteilungender Botanischen Staatssammlung München 12: 403-410.

GraU, J. 1987. Chromosomenzahlen chilenischer Mutisieen (Compositae). Botanischer Jahrbücher für Systematik, Pflanzengeschichte und Pflanzengeographie 108: 229-237.

Lovkvist, B. \& U. Hultgard. 1999. Chromosome numbers in south Swedish vascular plants. Opera Botanica 137: 1-42

Marticorena, C. 1990. Contribución a la estadística de la flora vascular de Chile. Gayana Botánica 47: 85-113.

Matthei, O. 1995. Manual de las malezas que crecen en Chile. Alfabeta impresores. Santiago, Chile. $545 \mathrm{pp}$.

Meerow, A. \& D. Snijman. 1998. Amaryllidaceae, en K. Kubitzki (ed.). The families and genera of 
vascular plants III. Flowering plants. Monocotyledons. Lilianae (except Orchidaceae), pp. 83-110. Springer. Berlin.

Morton, J. K. 1981. Chromosome numbers in Compositae from Canada and the USA. Botanical Journal of the Linnean Society 82: 357-368.

Naranjo, C. \& L. Poggio. 2000. Karyotypes of five Rhodophiala species (Amaryllidaceae). Boletín de la Sociedad Argentina de Botánica 35(3-4): 335-343.

Palma-Rojas, C. 2000. Caracterización citogenética de los géneros Rhodophiala Presl. y Phycella Lindl. (Amaryllidaceae), en P. Peñailillo \& F. Schiappacasse (eds). Los geófitos nativos y su importancia en la floricultura, pp. 73-79. Fundación para la Innovación Agraria (FIA) y Dirección de Investigación, Universidad de Talca (DIUT), Chile.

Powell, A., D. K yhos \& P. Raven. 1974. Chromosome numbers in Compositae. X. American Journal of Botany 61: 909-913.

Ravenna, P. 2003. Elucidation and systematics of the Chilean genera of Amaryllidaceae. Botanica Australis 2: 1-21.
Stephens, J., T. Tsuchiya \& H. Hughes. 1993. Chromosome studies in Alstroemeria pelegrina L. International Journal of Plant Science 154: 565-571.

Stuessy, T. \& C. TAYlor. 1995. Evolución de la flora chilena, en C. Marticorena \& R. Rodríguez (eds). Flora de Chile 1: 85-118.

Tsuchiya, T. \& A. Hang. 1989. Cytogenetics in the genus Alstroemeria. Herbertia 45: 163-170.

Weiss, H., T. Stuessy, J. Grau \& M. Baeza. 2003 a. Chromosome reports from South American Hypochaeris (Asteraceae). Annals of the Missouri Botanical Garden 90(1): 56-63.

Weiss, H., T. Stuessy, S. Siljak-Yakovlev, M. Baeza \& J. PARKER 2003b. Systematic and e volution ary implications of karyotypes of species of Hypochaeris (Asteraceae, Lactuceae) from South America. Plant Systematic and Evolution 241(3-4): 171-184.

Zhou, S., M. De Jeu, R. Visser. \& A. Kuipers. 2003. Characterisation of distant Alstroemeria hybrids: application of highly repetitive DNA sequences from A. ligtu subsp. ligtu. Annals of Applied Biology 142: 277-283.

Anexo 1. Procedencia del material.

Anexo 1. Material procedure.

Alstroemeria aurea Graham

VIII Región. Prov. Biobío: Parque Nacional Laguna del Laja sector Las Lagartijas, 1430 m, (37²2’S-71¹9'W). 8-I2003. C. Baeza 4201 (CONC). Salto de Las Chilcas, 1110 m, (37²2'S-71²4'W). 8-I-2003. C. Baeza 4202 (CONC). Prov. Nuble, cerca del Hotel Termas de Chillán, 1400 m, (36 53'S-71²5'W). 02-I-2003. C. Baeza 4190 (CONC). Termas de Chillán, en bosque de Nothofagus frente a las piscinas, 1609 m, (36 54 'S-71²4'W). 02-I-2003. C. Baeza 4193 (CONC). IX Región. Prov. Cautín: Victoria, road Curacautín to Victoria. 27-I-2004. K. Tremetsberger 1090 (CONC).

Alstroemeria hookeri Lodd. subsp. hookeri

VIII Región. Prov. Biobío: Puente El Roble, 64 m (36²5' S-72²5’W). 12-XII-2002. C. Baeza 4175 (CONC). Yumbel.

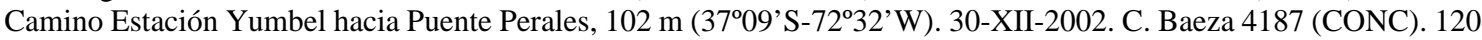

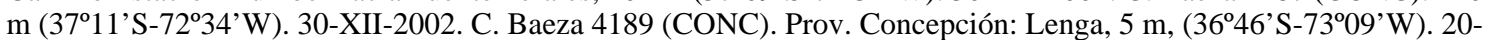
XII-2002. C. Baeza 4181 (CONC). Camino de San Pedro a Coronel, sector Stadio Italiano, 30 m (36º54'S-730' 'W). 20-XII-2002. C. Baeza 4182 (CONC).

Alstroemeria ligtu L.

VIII Región. Prov. Concepción: Bifurcación camino a Chome, 60 m (36²46'S-73¹1'W). 20-XII-2002. C. Baeza 4179 (CONC). Camino hacia Ramuntcho, $60 \mathrm{~m}\left(36^{\circ} 47^{\prime} \mathrm{S}-73^{\circ} 10^{\prime} \mathrm{W}\right)$. 20-XII-2002. C. Baeza 4178 (CONC). Camino a Caleta Lenga, ca. 500 mt, fábrica AGA, 20 m (3646'S-7307'W). 20-XII-2002. C. Baeza 4180 (CONC). Camino Concepción Santa Juana, cerca de Mitrinhue, 21 m (37 $7^{\circ} 00^{\prime} S-72^{\circ} 58^{\prime}$ W). 23-XII-2002. C. Baeza 4184 (CONC). Camino Concepción Santa Juana, km 17, 47 m (36 $58^{\prime}$ S-7300'W). 23-XII-2002. C. Baeza 4185 (CONC). Prov. Nuble: cerca del Hotel Termas de Chillán, 1340 m (3655’S-71²6’W). 02-I-2003. C. Baeza 4191 (CONC).

Alstroemeria pelegrina $\mathrm{L}$.

V Región. Prov. Valparaíso: Playa Quintay. 1997. INIA s/n (INIA). 
Alstroemeria presliana Herb. subsp. presliana

VIII Región. Prov. Ñuble: Termas de Chillán, ca. 500 mt, más arriba del Hotel Termas de Chillán, a 50 mt, de La Virgen, 1608 m (3654'S-71²4'W). 02-I-2003. C. Baeza 4192 (CONC).

Chaetanthera chilensis (Willd.) DC.

VIII Región. Prov. Ñuble: Camino entre Quillón y Cabrero, sector Canchilla, cerro con suelo arcilloso, 110 m (3652'S72²7’W). 14-I-2003. C. Baeza 4204 (CONC).

Chaetanthera ciliata Ruiz et Pav.

VIII Región. Prov. Ñuble: Camino entre Quillón y Cabrero, sector Canchilla, cerro con suelo arcilloso, $110 \mathrm{~m}$, (36 52'S72²7’W). 14-I-2003. C. Baeza 4205 (CONC).

Chaetanthera microphylla (Cass.) Hook. et Arn.

VIII Región. Prov. Ñuble: Puente El Roble, 64 m (36²45’S-72²5’W). 12-XII-2002. C. Baeza 4177 (CONC).

Chaetanthera moenchioides Less.

VIII Región. Prov. Biobío: Antuco, entre Villa Peluca y bifurcación a Rayenco, 710 m (37²0’S-71³5'W). 12-XII-2002. C. Baeza 4198 (CONC). Malalcura, lado de atrás del Hotel Malalcura, cerro de exposición norte, $950 \mathrm{~m}\left(37^{\circ} 22^{\prime} \mathrm{S}-\right.$ 71²9'W). 8-I-2003. C. Baeza 4199 (CONC). Prov. Ñuble: Quillón, Puente El Roble, 64 m (36²45'S-72²5’W). 12-XII2002. C. Baeza 4176 (CONC).

Grindelia anethifolia (Phil.) A.Bartoli \& Tortosa

VIII Región. Prov. Biobío: Yumbel. Camino Estación Yumbel hacia Puente Perales, 102 m (3709'S-72³2'W). 30-XII2002. C. Baeza 4186 (CONC). Yumbel. Camino Estación Yumbel hacia Puente Perales, $120 \mathrm{~m}\left(37^{\circ} 11^{\prime} \mathrm{S}-72^{\circ} 34^{\prime} \mathrm{W}\right)$. 30XII-2002. C. Baeza 4188 (CONC).

Grindelia prunelloides (Less.) A.Bartoli \& Tortosa

VIII Región. Prov. Biobío: Parque Nacional Laguna del Laja. Sector Los Barros, cerca del Refugio de los Militares, en una vega de Azorella trifurcata. 1411 m (37²27'S-71¹8'W). 9-I-2003. C. Baeza 4203 (CONC).

Haplopappus glutinosus Cass.

VIII Región. Prov. Biobío: Cruce camino Canteras a Antuco. 325 m (37²4’S-7200’W). 8-I-2003. C. Baeza 4197 (CONC). Prov. Concepción: Camino de San Pedro a Coronel, sector Stadio Italiano, $30 \mathrm{~m}$ (36 54'S-7308'W). 20-XII2002. C. Baeza 4183 (CONC).

Haplopappus grindelioides (Less.) DC.

VIII Región. Prov. Ñuble: Termas de Chillán, alrededores de las Termas 2000 m (3709’S-72³2’W). 30-XII-2002. P. López s.n. (CONC). Camino de Chillán a Yungay. 156 m (36²46’S-7208'W). 8-I-2003. C. Baeza 4195 (CONC).

Haplopappus macrocephalus (Poepp. ex Less.) DC.

VII Región. Prov. Linares: Termas de Catillo. 320 m (36¹7’S-71³8’W). 24-II-2006. C. Baeza 4258 (CONC).

Haplopappus stolpii Phil.

VIII Región. Prov. Ñuble: Quillón, Puente El Roble, 64 m (36²45’S-72²5’W). 14-I-2003. C. Baeza 4206 (CONC).

Hypochaeris acaulis (J.Remy) Britton

VIII Región. Prov. Biobio. Ralco. Reserva Forestal Biobio. Laguna La Mula, Pelada Mallín. 1706 m (3753’S-71²2’W). 31-I-2006. A. Terrab \& C. Baeza 20 (CONC).

Hypochaeris incana (Hook. et Arn.) Macloskie

XII Región. Prov. Tierra del Fuego. Primavera, sector Chillán. 100 m (5254’S-69²0’W). 8-II-2006. A. Terrab, C. Baeza \& E. Domínguez 52 (CONC).

Hypochaeris radicata $\mathrm{L}$.

VIII Región. Prov. Arauco. Entre Quidico y Tirúa. 30 m (3802’S-73²4’W). 24-III-2004. C. Baeza 4210 (CONC). Prov. Concepción. Camino de Coronel. Laguna La Posada. 25 m (3656'S-7309'W). 23-IV-2006. C. Baeza 4259 (CONC). 
Recuentos cromosómicos en plantas que crecen en Chile. III. BAEZA, M.

Hypochaeris scorzonerae (DC.) F.Muell.

V Región. Prov. de Valparaíso. Cuesta Balmaceda entre camino La Pólvora y Quebrada Honda, segunda curva desde la

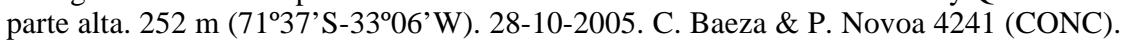

Leontodon autumnalis L.XII Región. Prov. Magallanes. Lago Blanco. Estancia Penitente. 247 m (5208' S-71²2 'W). 5II-2006. A. Terrab, C. Baeza \& E. Domínguez 39 (CONC).

Phycella bicolor (Ruiz et Pav.) Herb.

V Región. Prov. de Valparaíso. Jardín Botánico Nacional. 80 m (71³0’ S-3302' W). 18-X-2004. O. Fernández JBN 27 (INIA).

Phycella scarlatina Ravenna

IV Region. Prov. de Elqui. Camino al cerro Tololo, 26 km de la garita. 2025 m (7047’ S- 30¹0’ W). 11-VIII-2003. M. León \& M. Acosta BB 109 (INIA).

Placea amoena Phil.

IV Región. Prov. Limarí: Cuesta El Espino. 2001. Kew-INIA 013 (INIA).

Rhodophiala tiltilensis (Traub et Moldenke) Traub

Región Metropolitana. Prov. de Til Til. Caleu, fundo Lo Marín. 1114 m (7058’ S- 3259’ W). O. Fernández JBN 66 (INIA). 